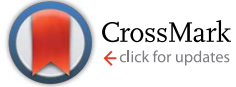

Cite this: RSC Adv., 2015, 5, 67308

\title{
Tooth lead levels as an estimate of lead body burden in rats following pre- and neonatal exposure
}

\begin{abstract}
Manish Arora*ab and Dominic J. Hare ${ }^{\text {acd }}$
Accurate assessment of early life lead exposure requires an accessible and reliable biomarker. Blood lead levels alone are not a suitable measure of chronic lead exposure, due to the rapid turnover and proportionally smaller amount compared to calcified tissues, such as bone and teeth. To further validate and standardise tooth lead levels as an appropriate biomarker of exposure, we designed a series of experiments where Wistar rats were fed, through drinking water, a regime of lead dosage that included stable, increasing and decreasing exposures, as well as prenatal exposure via the mother. At 15 days of age the animals were culled and blood, kidney, liver, brain, bone and teeth lead levels were assessed using inductively coupled plasma-mass spectrometry. Bone and tooth lead levels were by far the highest, and were reflective of the dosing parameters used. Brain lead levels were the lowest, suggesting that the blood-brain barrier provided some protection against lead uptake. These results give further support to the use of tooth lead levels as a biomarker of environmental lead exposure, particularly during early life periods.
\end{abstract}

Received 12th June 2015

Accepted 29th July 2015

DOI: 10.1039/c5ra11241a

www.rsc.org/advances

neurons. ${ }^{8}$ Long-term adverse health outcomes of children exposed to high levels of environmental lead include decreased intelligence quotient (IQ), poorer academic performance and delinquent behaviour. ${ }^{9}$ Studies in bacteria have shown that chronic lead exposure has significantly greater adverse effects on cell viability than acute toxicity, ${ }^{10}$ thus the persistency of lead in the environment is a pressing health concern.

The concept of critical windows of susceptibility to lead toxicity thus requires a better understanding of the differential lead body burden that is dependent on exposure timing. For instance, fetal exposure to lead during the first and second trimesters is associated with a higher risk of premature birth, ${ }^{\mathbf{1 1}}$ and prenatal lead exposure appears to increase the production of $\beta$-amyloid into young adulthood. ${ }^{12}$ Recent evidence has also shown that coexposure to lead with other potentially toxic metals, such as manganese during the second year of life can potentiate lead neurotoxicity. ${ }^{\mathbf{1 3}}$

The highly variable half-lives of lead stores in the body makes direct association of the timing and magnitude of exposure difficult to ascertain. Historically, much of our understanding of lead body burden comes from work performed in the mid-1900s, prior to the development of many of the more accurate analytical methods available today.

We have previously taken advantage of such methods, such as imaging by laser ablation-inductively coupled plasma-mass spectrometry to assess the metal levels within teeth. ${ }^{\mathbf{1 4 - 1 6}}$ Teeth are a particularly attractive biomarker of past chemical exposure, as they are able to exchange metal ions into the hydroxyapatite crystal structure and do not readily remobilise metals, ${ }^{17}$ 
as is the case with bone lead. Additionally, children's deciduous teeth can be collected with relative ease and in a non-invasive manner, and in combination with histological analysis; spatial information can be directly correlate with specific time periods of exposure.

In this work, we examined the distribution of lead in Wistar rats following pre- and postnatal lead exposure using inductively coupled plasma-mass spectrometry, with a particular focus on the uptake in teeth. The aim of these experiments was to investigate how differential timing of lead exposure during early life affected the distribution of the metal throughout the body, and if tooth lead concentrations provided an appropriate measure of lead body burden, to further support our efforts to develop a tooth-based biomarker of early life metal exposure.

\section{Experimental}

\section{Animals}

The experimental protocol used had the approval of the Animal Ethics Committee, Westmead Hospital, NSW, Australia (Ethics clearance no. 124.06). Ten inbred female Wistar rats, aged 1214 weeks, of normal weight (150-270 g) were divided into five groups of two, numbered Groups 1 to 5 , and mated with male Wistar rats, aged 12-14 weeks, of normal weight (200-400 g). The room housing the cages was kept at a temperature of 21-24 ${ }^{\circ} \mathrm{C}$ and humidity of between $40-60 \%$. A 12 hour light/dark cycle was employed, using an automatic timing device.

A total of 41 animals were raised for these experiments. Two rat pups belonging to Group 2 appeared to be rejected by the mother and showed physical signs of distress in the first week after birth, including low body temperature, wrinkled skin and low body weight. These animals were immediately culled. Body weights of all animals were within the reported normal range (5.0-6.4 $\mathrm{g}$ at birth $)^{18}$ and there were no significant differences in body weights between rat pups exposed to lead and those in the control group ( $p>0.05$; two-tailed Student's $t$-test). A total of 39 Wistar rat pups were included in the study ( $N$ per group: Group $1=11$; Group $2=5$; Group $3=7$, Group $4=7$; Group $5=9$ ).

\section{Lead administration protocols}

The animals were given ultrafiltered deionised water and fed a standard rodent diet (AIN93G, Glen Forrest Stockfeeders,
Western Australia) ad libitum which contained less than 0.006 $\mu \mathrm{g} \mathrm{g}^{-1}$ lead. Maternal rat exposure to lead nitrate (Asia Pacific Speciality Chemicals, NSW Australia) was via drinking water. The pups were housed with their mothers and were fed only by lactation and received no drinking water directly.

Details of the dose and timing of lead nitrate administration are given in Table 1. Group 1 served as a control and was not exposed to any lead nitrate. Group 2 received lead only during the prenatal period, while Groups 3 and 4 were given lead in an increasing and decreasing pattern respectively. Group 5 were given a fixed daily dose of lead during the experimental periods. These different patterns of lead exposure were used to mimic possible lead exposure events in a typical human environment. Drinking water bottles containing the ultrafiltered deionised water or lead nitrate were changed daily. The volume of water consumed was measured to determine the lead intake by the mother in each cage.

Exposure to lead nitrate occurred over three time periods, which included the prenatal period and two postnatal periods. The prenatal period of exposure started approximately five days before the birth of the pups, anticipating the commencement of crown formation in the first mandibular molar teeth approximately two days before birth. ${ }^{19}$ The margin of approximately three days was given to allow transplacental transfer of lead from the mother to the developing fetus. The postnatal period of exposure extended to 11 days after the birth of the pups, as the crown of the first mandibular molar tooth is completely formed at that time. ${ }^{20}$ The long postnatal period of tooth development provided the opportunity to administer two varying doses of lead nitrate. The postnatal period of lead exposure was divided into two time periods that extended from day-1 to day- 6 and from day-7 to day-11 respectively.

All the experimental animals were culled four days after the second postnatal period, on day-15. The designated doses of lead nitrate are not considered toxic and are substantially lower than the established fatal dose. ${ }^{21}$ Previous studies that have used similar or higher doses did not report any observable signs of acute toxicity. 22,23

\section{Tissue collection}

Pups were selected randomly from each of the five groups. Blood, organ, bone and tooth samples were harvested at appropriate time

Table 1 Concentration of lead nitrate in maternal drinking water and time periods of exposure for the five experimental groups of Wistar rats. Lead was administered via drinking water in one of five patterns: no lead (Group 1), lead only during the prenatal period (Group 2), an increasing dose (Group 3), a decreasing dose (Group 4) and a steady dose (Group 5). The doses were divided into three experimental time periods, one prenatal and two postnatal as indicated

\begin{tabular}{lllll}
\hline & \multicolumn{2}{l}{ Concentration of lead $\left(\mathrm{mg} \mathrm{L}^{-1}\right)$} & \\
\cline { 2 - 4 } Time period & $\begin{array}{l}\text { Group 1 no } \\
\text { lead }\end{array}$ & $\begin{array}{l}\text { Group 2 prenatal } \\
\text { dose }\end{array}$ & $\begin{array}{l}\text { Group 3 increasing } \\
\text { dose }\end{array}$ & $\begin{array}{l}\text { Group 4 decreasing } \\
\text { dose }\end{array}$ \\
\hline $\begin{array}{l}\text { Prenatal } \\
\begin{array}{l}\text { Day-5 to birth } \\
\text { Postnatal }\end{array}\end{array}$ & 0.00 & 40.00 & 10.00 & 40.00 \\
$\begin{array}{l}\text { Day 1 to day 6 } \\
\text { Day 7 to day 11 }\end{array}$ & 0.00 & 0.00 & 20.00 & 20.00 \\
dose
\end{tabular}


points as described here. Blood was taken from the maternal rats and pups by cardiac puncture immediately after euthanasia. The skin of the chest was cleaned using ultrafiltered deionised water and detergent (7X, ICN Biomedicals Inc., USA), and a sterile $2 \mathrm{~mL}$ syringe with a 25 gauge needle (Precision Glide, Becton Dickinson, Singapore) was used to withdraw blood from the heart. The collected blood was stored in sterile metal-free $2 \mathrm{~mL}$ tubes (EZ Micro Test Tubes, Bio-Rad Laboratories, USA) at $-20{ }^{\circ} \mathrm{C}$.

Following blood collection, the unerupted left and right mandibular first molar teeth, the liver, right kidney, brain and the right femoral bone were removed from each rat pup, using clean, sterile surgical instruments in a biological safety cabinet (Email Westinghouse Ltd NSW, Australia) to avoid any contamination from the laboratory environment. Organs were stored in sterile metal-free tubes (EZ Micro Test Tubes, Bio-Rad Laboratories, USA) at $-20{ }^{\circ} \mathrm{C}$. Analysis was restricted to first mandibular molar teeth, as these are the only molar teeth in Wistar rats that form enamel and dentine prenatally and can therefore be used to assess prenatal exposure to lead. ${ }^{\mathbf{1 4}}$

Molars were preferred over incisors in the present study because of the greater anatomical similarity between rodent and human molar teeth. Rat incisors lack enamel on the lingual aspect and do not have a root. ${ }^{19}$ In addition, an iron pigment is present on the surface of rat incisors, ${ }^{19}$ which may potentially affect the lead deposition in the enamel. Finally, the orientation of enamel rods in rat molar enamel is similar to that in humans, which is not the case in rat incisor enamel. ${ }^{24}$

\section{Inductively coupled plasma-mass spectrometry}

A Varian UltraMass-700 (Agilent, Australia) ICP-MS was used in this study. The instrument was calibrated using standards ranging in lead concentration from $2 \mathrm{ppb}$ to $500 \mathrm{ppb}$, made from a stock standard solution of $10 \mathrm{ppm}$ of lead (as nitrate) (Lot no. B1075023, Merck, Australia). The calibration curve was verified by analysing a certified reference material or quality control (QC) material that contained 80 ppb of lead (Merck, Australia). Bismuth-209 was used as an internal standard to correct for any drift in the observed lead counts, and lead-208 $\left({ }^{208} \mathrm{~Pb}\right)$ was the isotope monitored as the analyte. Instrument detection and quantification limits were determined using the standard $3 \sigma$ and $10 \sigma$ methods. ${ }^{25}$ Method detection limits (MDL) incorporating the dilution factor and any spectral interferences and drift were calculated using matrix matched standard reference materials, and varied from 0.005 to $0.02 \mu \mathrm{g} \mathrm{mL}{ }^{-1}$.

\section{Tooth and bone analysis by ICP-MS}

Whole left mandibular first molar teeth were cleaned mechanically under a dissecting microscope to remove any visible soft tissue. Samples were then placed in an ultrasonic bath of $5 \%$ $(\mathrm{v} / \mathrm{v})$ hydrogen peroxide for five minutes to further remove any adherent soft tissue. The samples were then treated in an ultrasonic bath of ultrafiltered deionised water for 10 minutes and dried at $60{ }^{\circ} \mathrm{C}$ for 2 hours. The samples were weighed using a calibrated digital balance and placed in a volumetric flask. $2 \mathrm{~mL}$ double-distilled ultrafiltered 65\% (v/v) nitric acid (Rhone Poulenc, France) was added to the flasks and the samples were left overnight for digestion. Sample solutions were then heated at $60{ }^{\circ} \mathrm{C}$ for up to 2 hours to ensure complete digestion and were diluted to $10 \mathrm{~mL}$ with ultrafiltered deionised water. The sample solutions were finally homogenised in an ultrasonic bath for $10 \mathrm{~min}$ and analysed by ICP-MS. NIST SRM-1486 (Bone Meal) (National Institute of Standards and Technology (NIST), Gaithersburg, MD, USA) was used as the external standard in each analytical run. One gram of the SRM-1486 was dissolved in $2 \mathrm{~mL}$ of double-distilled ultrapure nitric acid and processed as for teeth described above. Two to three standard solutions were analysed in each analytical run.

The analysis of the bone samples (right femurs) was essentially identical. Soft tissue was removed mechanically from the external surface of the bone sample. The samples were placed in an ultrasonic bath of $5 \%$ hydrogen peroxide for 5 minutes followed by cleaning in an ultrasonic bath of deionised water for 10 minutes. The samples were dried at $60{ }^{\circ} \mathrm{C}$ for 2 hours and dissolved in $2 \mathrm{~mL}$ of $65 \%$ nitric acid. The digest was subsequently diluted to $10 \mathrm{~mL}$ with ultrafiltered deionised water and analysed by ICP-MS. NIST SRM-1486 (Bone Meal) was used as the external reference standard for bone-lead analysis.

\section{Blood analysis by ICP-MS}

Whole blood samples were weighed using a calibrated digital balance before addition of $2 \mathrm{~mL} 65 \%$ nitric acid. Sample solutions were subsequently microwave-digested in Teflon containers (Ethos, Milestone Microwave Laboratory Systems, USA) for 10 minutes at $180{ }^{\circ} \mathrm{C}$. Samples were then diluted to $10 \mathrm{~mL}$ with ultrafiltered, deionised water.

More than $95 \%$ of the lead in blood is contained in the red blood cells, but it is the lead in plasma that exchanges with the different biological compartments. ${ }^{26}$ Lead concentrations in whole blood show strong positive correlations with plasma-lead levels. ${ }^{27}$ Whole blood was analysed as this allowed for the measurement of the total lead content in all fractions of blood and also facilitated easier detection of lead by making a larger concentration of lead available for measurement by ICP-MS.

Method blanks were prepared by diluting $2 \mathrm{~mL}$ of $65 \%$ nitric acid with deionised water to a final volume of $10 \mathrm{~mL}$. Method blanks were analysed in every run with five blanks for every 15-20 samples analysed. Matrix matched survey validated standard reference materials for blood, QC 410448 and QC02407 (Quality Control Technologies Pty. Ltd Australia) were analysed in each run. The standards were prepared in an identical manner to the blood samples.

\section{Organ analysis by ICP-MS}

Whole liver, kidney and brain samples were removed by dissection immediately after the animals were culled. Organs were stored in sterile metal-free $2 \mathrm{~mL}$ tubes (EZ Micro Test Tubes, Bio-Rad Laboratories, USA) at $-20{ }^{\circ} \mathrm{C}$. At the time of analysis, the organs were weighed individually, transferred to Teflon beakers and $2 \mathrm{~mL}$ each of $65 \%$ nitric acid and $30 \%$ hydrogen peroxide was added. The mixture was subjected to microwave digestion for 10 minutes at $180{ }^{\circ} \mathrm{C}$ and was subsequently diluted to $10 \mathrm{~mL}$ with deionised water. Sample 
solutions were homogenised in an ultrasonic bath for 10 minutes and analysed by ICP-MS.

Method blanks were prepared by diluting $2 \mathrm{~mL} \mathrm{65 \%} \mathrm{nitric}$ acid and $2 \mathrm{~mL} \mathrm{30 \%} \mathrm{hydrogen} \mathrm{peroxide} \mathrm{with} \mathrm{deionised} \mathrm{water} \mathrm{to}$ final volumes of $10 \mathrm{~mL}$. Method blanks were analysed in every run with approximately five blanks for every 15-20 samples analysed. The standard reference material NIST SRM-1577b (bovine liver) was used as an external standard. $2 \mathrm{~mL}$ each of nitric acid and hydrogen peroxide were added to $1 \mathrm{~g}$ of SRM$1577 \mathrm{~b}$. The solution was processed as for organs describe above. Two standard solutions were placed in each analytical run.

\section{Statistical analysis}

Lead levels in whole left first mandibular molar teeth of the rat pups were correlated with lead levels in the bone, kidney, liver and brain respectively using Pearson's correlation analysis. In addition, the Bland-Altman test was used to establish a quantitative relationship between lead levels in teeth, and the various organs studied. ${ }^{28}$ For the Bland-Altman test, logarithmic transformations were applied when comparing lead concentrations in teeth with lead levels in kidney, liver and brain, because the difference in lead concentrations between teeth and the organs increased with increasing tooth-lead levels. The KolmogorovSmirnov test was used to confirm the normal distribution of lead concentration in teeth, bone and key organs using SPSS version 11.0 (SPSS Inc., USA). Mean lead levels in tissue samples between groups were compared using an ordinary one-way ANOVA with a post hoc Tukey's multiple comparisons test. Outliers were identified as any variable falling outside two standard deviations of the mean and were excluded from the analysis.

\section{Results}

\section{Analytical performance}

Standard reference materials for blood (QC 410448 and QC 02407), organs (NIST SRM-1577b) as well as bone and teeth
(NIST SRM-1486), were analysed in each ICP-MS run. Percentage recovery of lead from these standards was within $7 \%$ of the expected value for blood and organs, and 5\% of the expected value for teeth (Table 2), confirming validity of ICP-MS analysis.

\section{Lead levels in blood, organs, teeth and bones}

Table 3 gives the total amount of lead consumed by maternal rats in each group and their mean blood-lead levels measured at the termination of the experiment. Group 5 had the highest mean maternal blood-lead levels, followed by Groups 3, 4 and 2 in decreasing order of mean blood-lead concentrations. This pattern reflects the amount of lead consumed by the respective maternal rats.

Fig. 1 shows mean lead levels in whole teeth, blood, bone and key organs (liver, kidney and brain) of the Wistar rat pups in Groups 1-5, measured by ICP-MS. Notably, teeth and bone had the highest concentrations of lead, while brain samples showed the lowest lead levels in every group.

Mean tooth-lead levels of the pups were significantly different between all five groups $(p<0.05)$ and showed the same pattern as the mean maternal blood-lead levels. Pups in Group 5 had the highest mean tooth-lead levels, followed by pups in Groups 3, 4 and 2 in decreasing order of mean tooth-lead concentrations. Additionally, the mean tooth-lead levels were generally ranked in the same order as the mean lead levels in the key organs in the exposure groups, with the one exception of the lead levels in livers of the pups in Group 3 which were lower than those of Group 2. Mean blood-lead levels of the pups also mirrored the maternal mean blood-lead levels, with only Groups 1 and 2; 2 and 4; and 3 and 5 showing no significant variation between experiments $(p>0.05)$. As expected, Groups 1 and 3 and 1 and 5 showed the most significant difference between mean blood lead levels ( $p<0.001)$.

The Pearson's correlation coefficient analysis of lead levels in the kidney, liver, brain and bone with lead concentrations in teeth and blood are given in Table 4 . When compared with

Table 2 Validity of analytical method. Two standard reference materials for blood, QC410448 (certified concentration of lead $=0.052 \pm 0.009$ $\left.\mu \mathrm{g} \mathrm{g}^{-1}\right)$ and QC02407 $\left(0.412 \pm 0.032 \mu \mathrm{g} \mathrm{g}^{-1}\right)$, an organ reference material, SRM-1577b $\left(0.129 \pm 0.004 \mu \mathrm{g} \mathrm{g} \mathrm{g}^{-1}\right)$ and a tooth and bone reference material, SRM-1486 $\left(1.335 \pm 0.014 \mu \mathrm{g} \mathrm{g}^{-1}\right)$ were analysed. All reported values are in $\mathrm{mg} \mathrm{g}^{-1}$ and \pm 1 standard deviation

\begin{tabular}{|c|c|c|c|c|c|c|c|}
\hline \multicolumn{2}{|l|}{ QC410448 (blood) } & \multicolumn{2}{|l|}{ QC02407 (blood) } & \multicolumn{2}{|l|}{ SRM-1577b (liver) } & \multicolumn{2}{|c|}{ SRM-1486 (bone meal) } \\
\hline $0.0480 \pm 0.0045$ & $96.53 \pm 1.61$ & $0.4180 \pm 0.0084$ & $102.6 \pm 2.3$ & $0.1230 \pm 0.0067$ & $98.47 \pm 4.80$ & $1.333 \pm 0.051$ & $100.2 \pm 3.8$ \\
\hline
\end{tabular}

${ }^{a} n=5 .{ }^{b} n=10 .{ }^{c} n=11$.

Table 3 Dose of administered lead and lead levels in maternal blood. Lead levels of blood from the two maternal rats in each group at the time of culling indicated that the amount of lead consumed reflected the total dose administered

\begin{tabular}{lllrr}
\hline & Group 1 & Group 2 & Group 3 & Group 4 \\
\hline Total maternal dose $(\mathrm{mg})$ & 0.00 & $1.68 \pm 0.28$ & $12.50 \pm 1.83$ & $7.41 \pm 0.41$ \\
Maternal blood-lead $\left(\mu \mathrm{g} \mathrm{g}^{-1}\right)$ & $0.01 \pm 0.02$ & $0.19 \pm 0.04$ & $0.36 \pm 0.03$ & $0.32 \pm 0.07$
\end{tabular}




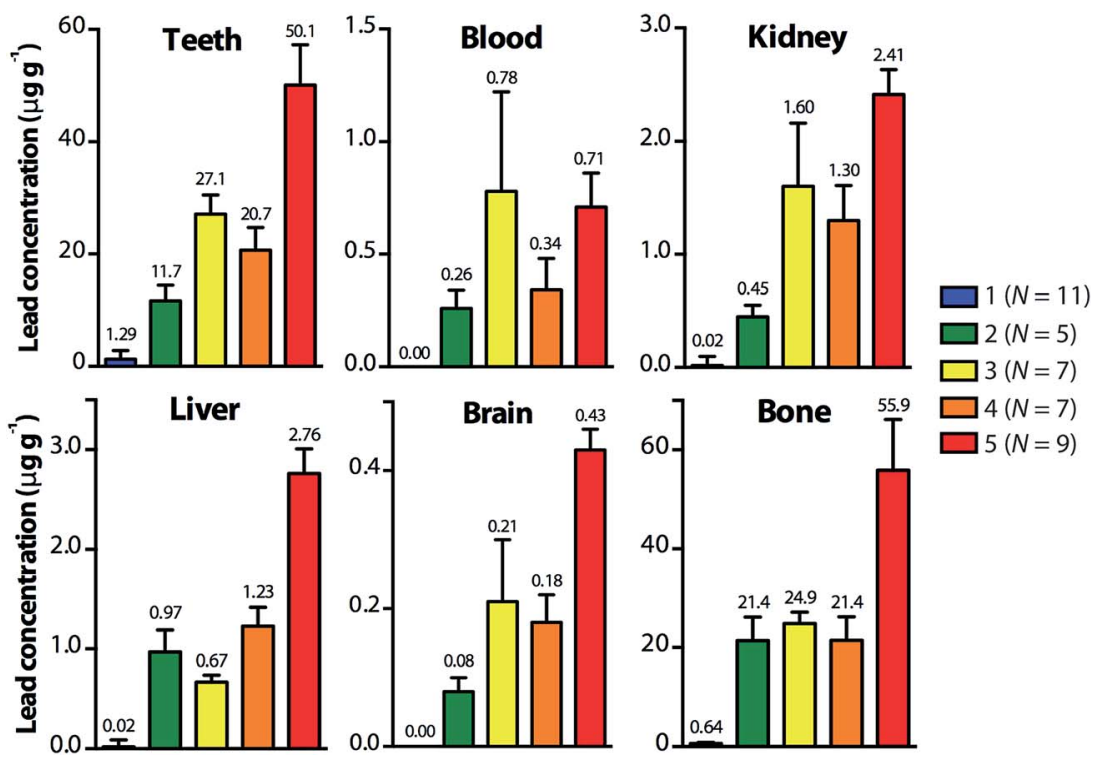

Fig. 1 Distribution of lead according to pre- and postnatal dosage regime. Concentrations of lead in each measured tissue type. Highest concentrations were observed in teeth and bone, with lowest concentrations found in the brain. Mean lead concentration $\left(\mu \mathrm{g} \mathrm{g}{ }^{-1}\right)$ are given for each measured sample.

Table 4 Correlation between tooth-, blood-, bone- and organ-lead data for rat pups in all groups. Lead concentrations in teeth and blood, were compared with lead concentrations in kidney, liver, brain and bone samples using Pearson's correlation coefficient analysis. Toothlead levels correlated more strongly with organs and bone-lead levels than blood-lead levels indicating that, in the present model, toothlead levels were a better predictor of body burden than blood-lead levels

\begin{tabular}{llll}
\hline Organ & Tooth-lead & Blood-lead & $p$ value \\
\hline Kidney $(n=38)$ & 0.82 & 0.73 & 0.35 \\
Liver $(n=37)$ & 0.90 & 0.38 & 0.001 \\
Brain $(n=34)$ & 0.91 & 0.60 & 0.001 \\
Bone $(n=38)$ & 0.89 & 0.56 & 0.001
\end{tabular}

Table 5 Bland-Altman analysis of the tooth-lead and organ-lead concentrations of the Wistar rat pups from the five experimental groups. Statistical analysis indicated that lead-levels in teeth were up to 79.6 times higher than lead levels in organs while bone-lead levels were more similar to tooth-lead levels

\begin{tabular}{lcl}
\hline Tooth-lead $v s$. organ-lead & Mean & $\begin{array}{l}\text { 95\% confidence } \\
\text { interval }\end{array}$ \\
\hline Tooth-lead : kidney-lead & 14.60 & $11.81,18.04$ \\
Tooth-lead : liver-lead & 22.81 & $18.36,28.34$ \\
Tooth-lead : brain-lead & 79.60 & $63.82,99.30$ \\
Tooth-lead minus bone-lead ${ }^{a}\left(\mu \mathrm{g} \mathrm{g}^{-1}\right)$ & -2.25 & $-5.40,0.88$
\end{tabular}

${ }^{a}$ For the Bland-Altman analysis, logarithmic transformations were applied when comparing lead concentrations in teeth, kidney, liver and brain. Consequently, results are reported as ratios of lead concentration in teeth and the respective organ. For comparisons between tooth-lead and bone-lead levels, logarithmic transformations were not required and results are reported as difference in mean lead concentration $\left(\mu \mathrm{g} \mathrm{g}^{-1}\right) .^{28}$ blood-lead, tooth-lead levels correlated more strongly with lead levels in each of the organs studied and this difference was statistically significant for liver, brain and bone $(p<0.05)$. The Bland-Altman analysis further allowed a quantitative relationship to be established between the tooth-lead levels and lead concentrations in the different organs studied (Table 5). With the exception of bone, lead levels in teeth were substantially higher than the lead concentrations of the other organs.

\section{Discussion}

The controlled exposure to lead of female Wistar rats and their pups in the present study has revealed a distinct pattern in the uptake of lead in teeth, blood, bone and the key organs studied. Lead uptake in the first mandibular molar teeth of the pups was substantially greater than blood, kidney, liver or brain samples (Fig. 1). Lead levels in teeth were between 34 to 70 times greater than the lead concentration in the blood of the pups. Additionally, the lead levels in teeth were of comparable magnitude to the lead levels in the femurs of the rat pups (Fig. 1). These results are similar to the findings of Grobler et al., ${ }^{29}$ who exposed rats to different levels of airborne lead, and found tooth-lead levels to be 57 to 250 times higher than blood-lead levels. They also found that lead levels in rat incisors were of similar magnitude to the lead concentrations in the alveolar bone. In the present study, kidney and liver samples showed higher lead levels than brain, similar to the results of Roels et al. ${ }^{23}$ who exposed lactating rats to different concentrations of lead in drinking water and measured the levels of lead in the liver, kidney, brain and heart of their sucklings.

Maternal blood-lead levels were highest in Group 5 followed by Groups 3, 4 and 2 in decreasing order of lead concentration. The tooth-lead and blood-lead levels of the pups closely reflected this pattern in maternal blood-lead levels, taken at the time 
of culling, and the total maternal intake of lead (Table 4). Notably, blood-lead levels of the pups were not significantly different between every group, while, the tooth-lead levels showed statistically significant differences between all groups. This outcome indicates that dental lead levels more accurately reflect the intensity of exposure which is most likely a consequence of the greater affinity of lead for calcified dental tissues coupled with a significantly longer period of retention of lead in teeth $^{30}$ as opposed to the relatively short half-life of lead in blood. ${ }^{4}$ Furthermore, the correlation analysis revealed that tooth-lead levels were significantly better predictors of lead levels in the bone, liver and brain than blood-lead levels in the present model. Both these facts support the proposition that dental lead levels are a reliable marker of exposure and body burden of lead, and that assessment of tooth-lead levels could be beneficial in childhood lead exposure studies, where blood data alone would be insufficient to accurately identify individuals at a health risk.

The results of the Bland-Altman analysis allowed quantitation of the relationship between tooth-lead levels and lead levels in key organs, which lends further support for the applicability of tooth-lead levels in the prediction of the body burden of lead. With further development of methods using prospective human studies, such as spatial analysis using laser ablation ICPMS, ${ }^{15,31,32}$ it may be possible to predict the concentration of lead in the key organs using tooth-lead levels.

Small amounts of lead were detected in samples from the control group (Group 1). Although the exact source of lead cannot be identified, it is believed that lead stores present in the maternal rats prior to commencement of the experiment may have been mobilised to blood during gestation and lactation, which is known to happen in humans, ${ }^{\mathbf{5}, 33}$ and this may explain the levels of lead found in the teeth, blood, bone and organs of the control rat pups. The levels of lead observed were, however, negligible and would not significantly influence the results of the present study.

Teeth have significant potential for use as a biomarker of early-life lead exposure. Though not as readily available as blood, saliva, or urine, they provide a retrospective assessment of temporal, rather than cumulative exposure. ${ }^{14}$ Nails and hair also provide some temporal information, though the nature of primary tooth formation and development presents a broader window extending from the second trimester to several years post-birth. ${ }^{34}$ When implemented into large-scale cohort studies, ${ }^{\mathbf{1 6}}$ deciduous teeth provide a non-invasive and easily collected resource for measuring neo, peri and postnatal metal exposure $^{35}$ that requires minimal handling and simple storage conditions. Discrete sampling using methods such as laser ablation-ICP-MS ${ }^{\mathbf{1 5}, 36}$ also permit repeated measurements of the sample with minimal destruction or sample disruption. Additionally, comparative measures of multiple deciduous teeth from the sample donor can be used to cross-validate and determine intra-sample variability with a high degree of accuracy and precision, which is not restricted to lead; complementary studies to the one presented here can also help further validate teeth as a biomarker of multiple metal exposures.

\section{Conclusions}

Teeth have been extensively used in the monitoring of environmental exposure to lead. However, very little is known about the correlations between dental lead levels and lead levels in key organs. In recognition of the potential limitations in using blood-lead levels alone for biological monitoring of childhood lead exposure, the development of a method for reproducible and reliable estimation of tooth-lead levels using primary human teeth becomes increasingly important. The present study has shown that a strong link exists between dental lead levels and lead levels in key organs, at doses between 10 and 40 $\mathrm{mg} \mathrm{L}^{-1}$, during the prenatal and neonatal periods in a rodent model. This protocol will potentially be of benefit in identifying individuals at health risk due to environmental lead exposure experienced during the prenatal and neonatal periods of development. Additionally, this further supports our previous use of this method to study of the long-term health effects of in utero lead exposure, which has previously been restricted due to the absence of a suitable biomarker for prenatal lead exposure.

\section{References}

1 G. Flora, D. Gupta and A. Tiwari, Interdiscip. Toxicol., 2012, 5, 47-58.

2 H. Needleman, Annu. Rev. Med., 2004, 55, 209-222.

3 P.-C. Hsu and Y. L. Guo, Toxicology, 2002, 180, 33-44.

4 S. K. Karri, R. B. Saper and S. N. Kales, Curr. Drug Saf., 2008, 3, 54-59.

5 E. K. Silbergeld, Environ. Health Perspect., 1991, 91, 63-70.

6 D. C. Basha, M. U. Rani, C. B. Devi, M. R. Kumar and G. R. Reddy, Int. J. Dev. Neurosci., 2012, 30, 343-350.

7 C. Nava-Ruiz, M. Méndez-Armenta and C. Rios, J. Mol. Histol., 2012, 43, 553-563.

8 X. Lu, C. Jin, J. Yang, Q. Liu, S. Wu, D. Li, Y. Guan and Y. Cai, Biol. Trace Elem. Res., 2012, 151, 75-84.

9 P. Grandjean and P. J. Landrigan, Lancet Neurol., 2014, 13, 330-338.

10 L. Yuan, W. Zhi, Y. Liu, S. Karyala, P. J. Vikesland, X. Chen and H. Zhang, Environ. Sci. Technol., 2015, 49, 824-830.

11 D. Cantonwine, H. Hu, B. N. Sánchez, H. Lamadrid-Figueroa, D. Smith, A. S. Ettinger, A. Mercado-García, M. HernándezÁvila, R. O. Wright and M. M. Tellez-Rojo, J. Occup. Environ. Med., 2010, 52, 1106-1111.

12 M. Mazumdar, W. Xia, O. Hofmann, M. Gregas, S. Ho Sui, W. Hide, T. Yang, H. L. Needleman and D. C. Bellinger, Environ. Health Perspect., 2012, 120, 702-707.

13 B. C. Henn, L. Schnaas, H. Lamadrid-Figueroa, M. Hernández-Ávila, H. Hu, M. M. Tellez-Rojo, A. S. Ettinger, J. D. Schwartz, C. J. Amarasiriwardena, D. C. Bellinger and R. O. Wright, Environ. Health Perspect., 2012, 120, 126-131.

14 M. Arora and C. Austin, Curr. Opin. Pediatr., 2013, 25, 261267.

15 D. Hare, C. Austin, P. Doble and M. Arora, J. Dent., 2011, 39, 397-403. 
16 R. B. Gunier, A. Bradman, M. Jerrett, D. R. Smith, K. G. Harley, C. Austin, M. Vedar, M. Arora and B. Eskenazi, Environ. Sci. Technol., 2013, 47(19), 1124911257.

17 A. Steenhout, Arch. Environ. Health, 1982, 37, 224-231.

18 D. Pass and G. Freeth, ANZCCART News, 1993, 6, 1-4.

19 B. K. Berkovitz, G. R. Holland and B. J. Moxham, Color Atlas \& Textbook of Oral Anatomy, Histology, and Embryology, Mosby Inc, 1992.

20 M. M. Hoffman and I. Schour, Am. J. Orthod. Oral Surg., 1940, 26, 854-874.

21 N. I. Sax and R. J. Lewis, Dangerous Propoerties of Industrial Materials, Van Nostrand Reinhold, New York, 7th edn, 1989.

22 R. K. Dearth, J. K. Hiney, V. Srivastava, S. B. Burdick, G. R. Bratton and W. L. Dees, Reprod. Toxicol., 2002, 16, 343-352.

23 H. Roels, R. Lauwerys, J.-P. Buchet and G. Hubermont, Toxicology, 1977, 8, 107-113.

24 Z. Skobe and D. Stern, Arch. Oral Biol., 1978, 23, 307-316.

25 J. M. Green, Anal. Chem., 1996, 68, 305A-309A.

26 R. W. Baloh, Arch. Environ. Health, 1974, 28, 198-208.
27 P. Apostoli, J. Chromatogr. B: Anal. Technol. Biomed. Life Sci., 2002, 778, 63-97.

28 J. M. Bland and D. G. Altman, Stat. Methods Med. Res., 1999, 8, 135-160.

29 S. R. Grobler, R. J. Rossouw, T. J. v. W. Kotze and I. A. Stander, Arch. Oral Biol., 1991, 36, 357-360.

30 S. Skerfving, U. Nilsson, A. Schütz and L. Gerhardsson, Scand. J. Work, Environ. Health, 1993, 19, 59-64.

31 M. Arora, D. Hare, C. Austin, D. R. Smith and P. Doble, Sci. Total Environ., 2011, 409, 1315-1319.

32 M. Arora, C. Austin, B. Sarrafpour, M. Hernández-Ávila, H. Hu, R. O. Wright and M. M. Tellez-Rojo, PLoS One, 2014, 9, e97805.

33 B. L. Gulson, C. W. Jameson, K. R. Mahaffey, K. J. Mizon, M. J. Korsch and G. Vimpani, J. Lab. Clin. Med., 1997, 130, 51-62.

34 M. M. Ash and S. J. Nelson, Wheeler's Dental Anatomy, Physiology and Occlusion, WB Saunders, Philadelphia, 2003.

35 F. Barbosa Jr, J. E. Tanus-Santos, R. F. Gerlach and P. J. Parsons, Environ. Health Perspect., 2005, 113, 1669-1674.

36 D. Kang, D. Amarasiriwardena and A. H. Goodman, Anal. Bioanal. Chem., 2004, 378, 1608-1615. 